\title{
Dynamic Evolution of Service Trade Network Structure and Influence Mechanism in Countries along the "Belt and Road" with Big Data Analysis
}

\author{
Yang Liu ${ }^{1}{ }^{1}$ and Sang-Bing Tsai ${ }^{2}{ }^{2}$ \\ ${ }^{1}$ School of Business and Economics, Shandong Management University, Jinan, Shandong 250000, China \\ ${ }^{2}$ Regional Green Economy Development Research Center, School of Business, WUYI University, Nanping, China
}

Correspondence should be addressed to Yang Liu; liuyang6494@126.com

Received 5 November 2021; Revised 7 December 2021; Accepted 14 December 2021; Published 3 January 2022

Academic Editor: Liu Zhi

Copyright (c) 2022 Yang Liu and Sang-Bing Tsai. This is an open access article distributed under the Creative Commons Attribution License, which permits unrestricted use, distribution, and reproduction in any medium, provided the original work is properly cited.

\begin{abstract}
With the accelerating process of economic globalization, the deep adjustment of the international industrial division of labor, and the continuous upgrading of the global value chain, the development of the service industry has entered into a new historical period. This study analyzes the dynamic evolution of trade network structure at three levels: macro, mesa, and micro, and empirically explores its influencing factors. Taking national service trade as the research object, this study analyzes the topological structural characteristics of the food trade network of "One Belt and One Road" countries by selecting indicators such as network point degree, average path length, trade intensity, intermediation degree, and aggregation coefficient, and further explores the characteristics of association network structure in national service trade network using block model. The topological structural characteristics of the "Belt and Road" trade network are analyzed from three aspects: overall, local, and individual. Then, we construct a regression model to analyze the factors influencing the dynamic evolution of the service trade network structure of the countries along the "Belt and Road" through the QAP method. Finally, based on the summary of the research findings, this study puts forward the corresponding considerations and policy recommendations from the perspectives of regional, directional, and reciprocal investment, to benefit more efficient and strategic national service trade under the framework of "Belt and Road." Based on the results of this study, this study proposes the following policy recommendations: firstly, actively promote the service trade cooperation with other countries to optimize the market layout; secondly, promote the service industry reform to extend the length of the value chain and improve its position in the value chain; and thirdly, jointly promote the infrastructure construction of "Belt and Road" with other countries, promote trade liberalization, and reduce the trade volume. Secondly, we should promote the reform of the service industry, extend the length of the value chain, and improve our position in the value chain. Finally, we should vigorously promote the development of innovation, promote the two-way opening of new service industries, adhere to the "going out" strategy, and cultivate high-quality and excellent technical talents, to promote the overall improvement of the innovation-driven capacity of the service industry with a people-oriented approach.
\end{abstract}

\section{Introduction}

At present, the world economy has experienced a weak recovery after the financial crisis and the rise of trade protectionism, and how to solve the abovementioned problems in the new important historical period and maintain medium- and high-speed economic development has become the top priority of economic construction. The
"Belt and Road" Summit Forum marks the beginning of the "Belt and Road" initiative into a new stage of history, mainly for optimization and upgrading [1]. The "Belt and Road" initiative provides a new recipe for economic development and the recovery of the world economy during the "13th Five-Year Plan" period and with the advancement of the "Belt and Road" construction. It will also promote the rapid development of the service industry. At this stage, the world 
economy is still in shock and slowly recovering, and cooperation among many regional organizations is still flourishing $[2,3]$. The "Belt and Road" project requires the use of multilateral cooperation mechanisms to promote the construction and sustainable development of the region. We construct the trade network structure of "Belt and Road" and analyze the characteristics and evolution of trade relations among countries in the network. Based on theoretical research, we analyze the trade network model of "Belt and Road" based on relationship attributes, combine the relevant indicators of social network analysis to explore the trade relations of countries in-depth, and use software to form trade visualization diagrams to visually show the basic form of "Belt and Road" trade network [4]. The trade visualization diagram is formed using software to visualize the basic form of the "Belt and Road" trade network, the overall network attributes, and the microdevelopment of each country. The research combines international trade with network science, builds an interdisciplinary theoretical analysis framework, and uses a network dynamic model based on the perspective of the formation of trade relations to explore the formation mechanism and pattern of service trade in countries along the "Belt and Road" [5]. The influence mechanism can provide more scientific and reasonable explanations and suggestions for the formation of deep integration in the global trading system.

Since the implementation of the "Belt and Road" strategy, the changes in the pattern of service trade in the countries along the route can deepen cooperation and exchanges with countries along the route, win-win, and development, and facilitate the further acceleration of the pace of trade cooperation with countries along the route. In the context of economic globalization, regional economic development affects the level of world economic development. Studying the overall network structure of horticultural product trade in countries along the route will respond to the challenges of economic globalization and seize the opportunities brought by globalization that has long-term practical significance [6]. This study focuses on the structural and relational dimensions of social networks to explore the national trade relations in the trade network of "One Belt, One Figured Road," to measure the relative position of countries in the trade network of "One Belt, One Figured Road," and to analyze the relative position of countries in the trade network of "One Belt, One Figured Road." This study analyzes the structure of subgroups in the trade network of "Belt and Road," which grasps the trade of core countries and drives the economic linkage of neighboring countries, to obtain better development and achieve better innovation. This study analyzes the core countries in the trade network structure, which have an important influence on the trade network, and discusses the degree and scope of influence of the core countries on the subordinate countries [7]. This study has a more comprehensive understanding of the trade of agricultural products among countries along the route. It thoroughly analyzes the internal characteristics and evolutionary laws of the trade network structure and then analyzes the various influencing factors that affect the trade network structure, so that the changes in the trade network structure can be judged more clearly. The integration process will help to find countermeasures and suggestions to promote the sustainable development of agricultural trade in the "Belt and Road" initiative.

Through an in-depth analysis of the dynamic evolution of service trade network structure and influence mechanism in countries along the "Belt and Road," this study can provide an important reference for decision-making on where and how to integrate the service industry into the global trade system; at the same time, it will give reasonable and effective policy suggestions and strategies on how to enhance the influence of service trade in key regions. The first section is the introduction, which mainly describes the background and significance of this study, as well as the main research content, framework, methodology, and innovation points of this study. The second section is the related work of this study, which is a comprehensive review of the existing literature from three levels: the current situation of international trade in service research, international trade research from the perspective of complex networks, and international trade network research in the context of the global value chain. The third section is a study on the dynamic evolution of service trade network structure and influence mechanism in countries along the "Belt and Road," from the indexes of service trade in countries along the "Belt and Road," the construction of the dynamic evolution model of network structure, and the study of service trade in countries along the "Belt and Road," respectively. The study is carried out in three aspects: indicators of service trade of countries along the "Belt and Road," model construction of network structure dynamic evolution, and influence mechanism of service trade of countries along the "Belt and Road." The fourth section is the analysis of the results. The author selects population size, free trade agreements, geographical distance, common language, and economic scale as the main variables to be included in the model and uses the quadratic assignment procedure (QAP) method to analyze the correlation and regression of relevant influencing factors and the value-added trade network of service industries along the "Belt and Road." The fifth section is the last chapter of this study. Chapter 5 is the last chapter of this study, which provides a comprehensive summary and discussion on how to strengthen service trade cooperation with countries along the "Belt and Road" in the new period and new situation, promote the continuous and steady development of the "Belt and Road" initiative, promote the transformation and upgrading of the domestic service industry, and comprehensively improve the service industry.

\section{Related Work}

Since the trade network can be effectively interpreted and measured at the level of regional organizations through the complex network social structure, scholars at home and abroad began to widely study and use the complex social network idea in regional international trade research, in which the overall structural characteristics of the regional organization's foreign trade-economic network (including 
centrality, central potential, and shortest distance) were effectively measured and identified, and scholars modeled and analyzed. The probability network is used to empirically analyze the formation mechanism of real economic trade networks [8]. Teo et al. analyzed the spatial and temporal factors and structural characteristics of the industrial development of the five Central Asian countries on the Silk Road Economic Belt using statistical methods with mathematical and spatial ideas [9]. Dumor et al. used the exponential random graph model (ERGM) in the social network analysis method to identify and measure the overall pattern and local characteristics of manufacturing trade in the countries along the "Belt and Road" from the overall and local perspectives, and empirically analyzed the national trade system in terms of religion and culture, language interoperability, national GDP, R\&D investment, and the government's foreign trade system [10]. We also analyze the impact of national attributes such as religion and culture, language sharing, national GDP, R\&D investment, and government foreign trade system on the economic network of the manufacturing industry along with the "Belt and Road." Summers analyzed the comparison and development trend of labor-intensive and capital-intensive product trade in the bilateral trade structure, and the results showed that the trade development is not balanced, and the long-term development and importance of foreign trade make it stronger than Vietnam in the scale and growth efficiency of export trade, while Vietnam is more prominent in the promotion of product categories and the restructuring of export trade through labor security [11]. For example, the labor force has a certain advantage for both countries' trade development, and the advantageous effect is diminishing, and both countries are facing the bottleneck of technology development [12].

Complex network theory is the theoretical foundation of the overall network analysis method, which is a relationship analysis method with holistic and global characteristics, and can show the overall network structure, density, and the relationship between the overall network members, and is a complex social network analysis method. The trade network structure also shows the scale-free, small-world, and high aggregation of the complex network, and countries with a high number of points in the trade network are more likely to have trade relations with countries with a low number of points [13]. Based on the trade data of the countries along the "Belt and Road," Cui and Song analyzed the trade network structure from the perspective of centrality and block model and used QAP correlation analysis and regression analysis to analyze the factors influencing the weighted trade network structure of the countries along the "Belt and Road," pointing out that institutional factors are the main factors influencing the trade network structure [14]. Lee et al. constructed natural gas networks in the countries along the route, measured natural gas network density, centrality, and heterogeneity, and used QAP regression analysis to compare and analyze the influencing factors of unweighted and weighted natural gas network structures in the countries along the route, and the results showed that population size and urbanization level had significant effects in the unweighted regression analysis [15]. $\mathrm{Li}$ et al. analyzed the density, centrality, and core edge of the service trade pattern of the "Belt and Road" countries and pointed out the core position in the service trade of the "Belt and Road" countries. The research results of using the overall network analysis method to analyze the trade network structure of the countries along the route and its influencing factors have been increasing, which provides a good reference for this study [16]. However, the research on national service trade among the countries along the route is mainly based on the analysis of trade potential and trade efficiency using the parameter estimation method such as the trade gravity model, while some scholars use the social network analysis method to analyze the service trade network structure of the countries along the route [17-19].

This article focuses on examining the international investment network of countries along the "Belt and Road" and grasps this important topic as a whole. At the same time, in the empirical test part, this article discusses the characteristics of the country in the network and the characteristics of the overall network and examines the status of a country in the international investment network along the "Belt and Road" and the international investment network itself from the economic, political, and geographic dimensions [20,21]. However, as the fastest-growing emerging country in the "Belt and Road" trade network, it influences the trade structure and trade industry of Central and South Asia, and its position in the "Belt and Road" trade network should not be underestimated. In international trade, the country's trade status is based on a relationship with other countries, which is the relationship property of the country in the world. Previously, scholars mainly analyzed the trade volume and trade structure, and studied the country's individual property, but lacked the sociological basis of relationship analysis [19]. In this study, we analyze the economic activities of the countries in the "Belt and Road" trade structure through the trade relations among the countries in the "Belt and Road" construction structure and combine the social network analysis method to quantitatively compare the degree of international participation of different countries and the international formation of economic networks among countries. Constructing the "Belt and Road" service industry value-added trade network from the network perspective, showing the "Belt and Road" service industry value-added trade and service industry value-added trade in the form of a network, will help to vividly analyze the international status of service trade and the "Belt and Road" "One Road" market layout.

\section{Research on the Dynamic Evolution of Service Trade Network Structure and Influence Mechanism of Countries along the "Belt and Road"}

3.1. Research on Service Trade Indicators of Countries along the "Belt and Road". The overall network is a collection of overall relationships, which is simply described as a combination of nodes and edges. Nodes can be countries, cities, 
people, groups, cells, etc. The data conforming to each node constitute edges, and edges connect each node to represent the relationships between each other. The overall trade network is a collection of overall trade relationships, with traders as nodes and trade relationships between countries as edges. Trade relations can be divided into import and export, reflecting the directionality of the edge; there is a quantitative distinction between import and export trade relations of each country, indicating that the edge has weight. In this way, the overall trade network can be divided into unweighted undirected, unweighted directed, weighted undirected, and weighted directed networks. The overall network of service trade of the countries along the route is a collection of trade relations of the countries along the route, and a single country is a node.

The network density reflects the tightness and relevance of the connection of each country in the overall network; the higher the density, the more closely connected the overall network is, the greater the influence on the overall network, and the more beneficial to the resource sharing among nodes $[22,23]$. The overall network density with directional weighting for trade in services of the countries along the route can be expressed by equation (1). $N(d)$ denotes the network density, AR denotes the actual number of relationships between the nodes of the overall network, and $\mathrm{N}$ denotes the number of nodes in the overall network.

$$
\begin{aligned}
& N(d)=\frac{\mathrm{AR}}{N *(N-1)}, \\
& N(d) \subseteq[0,1] .
\end{aligned}
$$

Reciprocity reflects the mutuality of the relationship between nodes and reflects the degree of mutual choice between nodes. The greater the reciprocity, the faster the dissemination and flow of trade information between nodes, the more orderly trade cooperation, and the stabilization of the overall trade network structure. The average distance refers to the average "neighboring" distance between nodes in the overall network, reflecting the accessibility between nodes; the shorter the average distance, the stronger the accessibility between two nodes. Cohesiveness also reflects the degree of connection between nodes, and unlike other indicators, cohesiveness reflects the degree of connection between every three nodes, and there is a network relationship between them.

Degree centrality is divided into outgoing degree centrality and incoming degree centrality, and outgoing degree centrality and incoming degree centrality reflect the ability of a node in the network to send out or receive other nodes in the overall network of service trade of the countries along the route. The calculation method to represent outgoing centrality and incoming centrality is shown in equation (2), where $O(m)$ denotes the outgoing centrality of node $m, I(m)$ denotes incoming centrality, $\sum N_{m n}$ denotes the number of nodes pointing to other nodes, and $\sum N_{n m}$ denotes the number of nodes accepting the number of other nodes.

$$
\left\{\begin{array}{l}
O(m)=\frac{\sum_{n=1}^{N} N_{m n}}{(N-1)}, \\
I(m)=\frac{\sum_{n=1}^{N} N_{n m}}{(N-1)} .
\end{array}\right.
$$

Intermediary centrality refers to the shortcut of a node over all nodes of the overall network, reflecting the node's ability to control resources, which is expressed in equation (3), where $C(m)$ denotes intermediary centrality, $d(n m)_{m}$ is the set of all minimal paths connecting node $n$ and node $m$ through $m$, and $d(n m)$ is the set of all minimal paths connecting node $n$ to node $m$.

$$
C(m)=\sum_{n=1}^{N} \sum_{m=1}^{N} \frac{d(n m)_{m}}{d(n m)}
$$

Proximity centrality reflects the shortest distance between nodes, and the sum of the distances from a node to all nodes in the overall network is the largest, and the larger the proximity centrality, the shorter the path for that node to access resources and information. Proximity centrality can be divided into outgoing proximity centrality and incoming proximity centrality, and its calculation formula is given in equation (4), where $O(m)$ denotes outgoing proximity centrality, $I(m)$ denotes incoming proximity centrality, $d(n m)$ denotes the shortest path from node $m$ to node $n$, and $d(m n)$ denotes the shortest path from node $n$ to node $m$.

$$
\left\{\begin{array}{l}
O(m)^{-1}=\sum_{n=1, n \neq m}^{N} d(m n), \\
I(m)^{-1}=\sum_{n=1, n \neq m}^{N} d(n m) .
\end{array}\right.
$$

The measures of unweighted networks contain network density, degree centrality, medium reading, and aggregation coefficient. This study extracts the trade data between countries along the "Belt and Road" from the United Nations Commodity Trade Database and builds a trade matrix. Through the annual trade matrix, a network snapshot of the international trade relationship network can be topped up, and then, the dynamic evolution and analysis of the annual trade situation are carried out.

3.2. Network Structure Dynamic Evolution Model Construction. To maintain the integrity and data accessibility of "Belt and Road," the national service trade data of all countries along the route are selected as the basis for constructing the trade network. According to the theory of complex networks, the trade network is constructed with each country as a node, the trade relationship between countries as an edge, and the total amount of national service trade as the weight of the edge. Since trade flow direction is not considered, this study constructs the unweighted undirected network and the weighted undirected network, respectively. The dynamic evolution model of the network structure is shown in Figure 1. In the network, some nodes will be in a more important position because they are more 


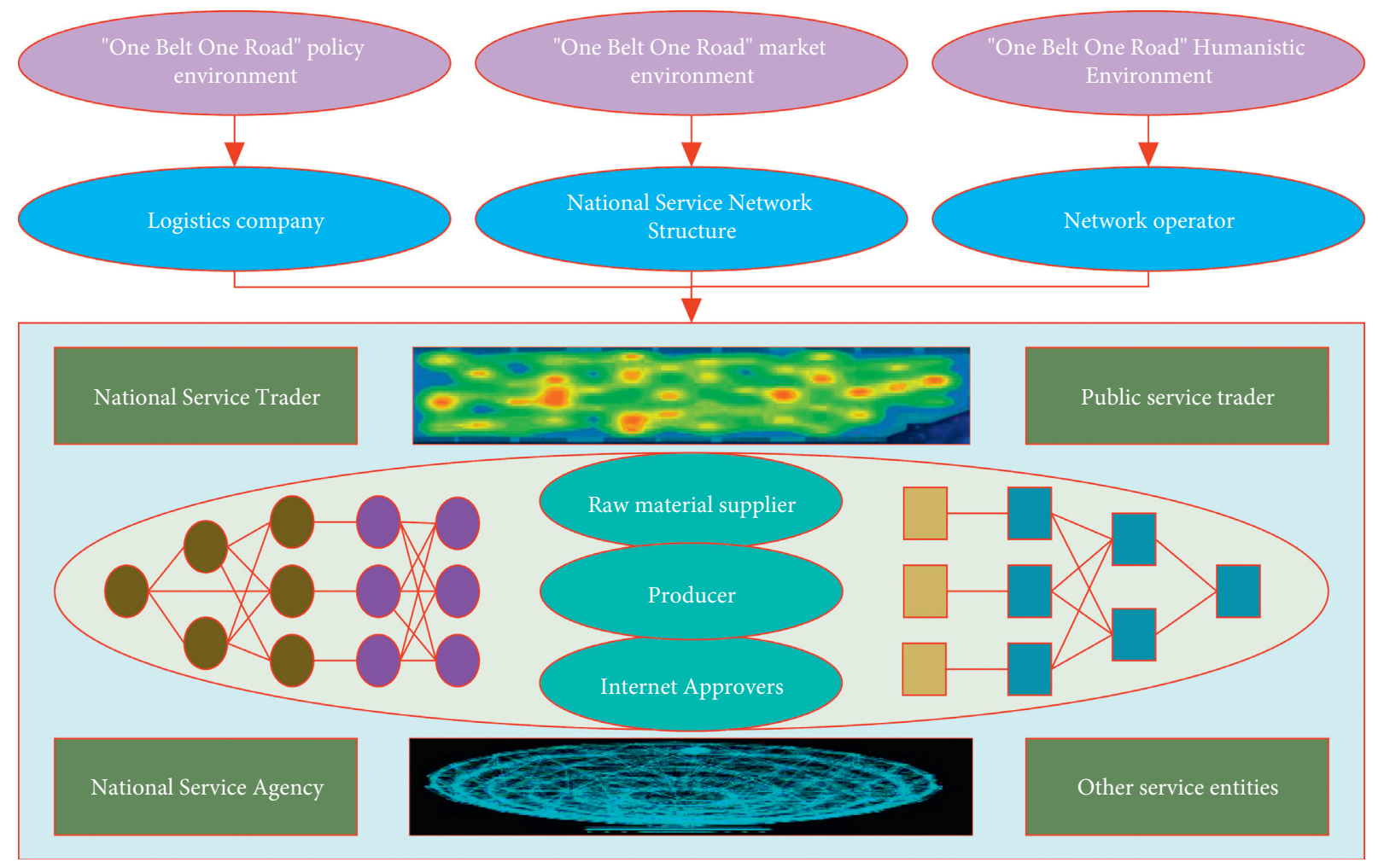

FIGURE 1: Dynamic evolutionary model of network structure.

connected with other nodes and have a stronger role, thus forming a closely connected core group. At the same time, some nodes will also form a fringe group at the periphery of the network because they are less connected to other nodes and have less influence in the network. The two-way trade segment has strong and export-oriented trade ties with other segments, while the trade ties between countries within the segment are also strong. The inward trade segment's trade relations occur mainly within the segment, and the trade links with other segments are less and mainly import. The outward trade segment has closer trade ties with other segments and is mainly export-oriented compared with the intraplate segment. The isolated trade segment not only has fewer trade ties among countries within the segment, but also has few trade ties with other segments, and is therefore at the edge of the network (see Figure 1).

To more accurately describe the overall structural characteristics of the value-added trade networks of service industries along with the "Belt and Road," this study analyzes the complete networks of TEX, DVA, and FVA in four aspects: the number of connected edges, the average path length, the network density, and the agglomeration coefficient. The number of network edges $M$ denotes the number of relations directly connected by each node in the network, which is shown in equation (5), where $\mathrm{N}$ represents the number of countries in the network (same below), $b_{m n}$ is the element in the corresponding unweighted adjacency matrix in the network, $b_{m n}=1$ indicates the presence of directed edges pointing from node $m$ to node $n$, and $b_{m n}=0$ indicates the absence of directed edges pointing from node $m$ to node $n$. A higher number of connected edges indicates that the number of trade relations in the network is higher.

$$
A R=\sum_{m, n=1}^{N} b_{m n} .
$$

The average path length of the network is used to portray the average number of nodes to be experienced by one node to another node in the network and is used to measure the effectiveness of information transfer between network nodes, and in this study, the average path length is used to measure the accessibility of trade relations between countries; see equation (6), where $d(m n)$ denotes the shortest distance between node $m$ and node $n$, the minimum number of intermediate nodes through which node $m$ and node $n$ are connected. The shorter the average road length, the more efficient the information transfer in the network and the more convenient the trade exchange.

$$
\mathrm{APL}=\frac{\sum_{m, n=1}^{N} d(m n)}{N *(N-1)} * 100 \% .
$$

The clustering coefficient is used to portray the trade clustering effect of a network and is an important indicator of the existence and closeness of the association between other nodes connected to a node. The clustering coefficient is calculated in two ways, one is based on the average local density. The clustering coefficient of the whole network is equal to the mean value of the individual point density coefficients, as in equation (7), where $\beta_{k}$ is the number of trade relations in country $k, p_{k}$ is the frequency of trade relations existing between any two trading partners of country $k$, and $\mathrm{N}$ is the trade network node. 


$$
f(x)=\sum_{k=1}^{N} \frac{p_{k}}{N * \beta_{k} *\left(\beta_{k}-1\right)} .
$$

In network analysis, the centrality of a node in a network is not only related to the number of points it is connected to, but also related to the importance of its connected nodes in the network. In general, the importance of a node increases with the relationship between the node and other important nodes, and this importance is measured by the eigenvector centrality. The formula for calculating the feature vector centrality is shown in equation (8), where $\beta$ and $h(n)$ are the maximum eigenvalues of the adjacency matrix and its eigenvectors, and $d(m n)$ indicates the existence of relationships between nodes.

$$
H(m)=\beta^{-1} * \sum_{n=1}^{N} d(m n) * h(n) .
$$

Unauthorized network QAP regression helps to analyze the factors affecting investment linkages between countries in the area along the "Belt and Road," while empowered network QAP regression helps analyze the influencing factors of the amount of investment linkages between countries. The combination of QAP analysis of the power network will better explain the deep-seated reasons for the formation, evolution, and development of the international investment network between China and the countries along the "Belt and Road."

\subsection{The Impact Mechanism of Service Trade in Countries along} the "Belt and Road". In this study, we decided to select the factors influencing the change in trade network structure in four aspects: economic, geographical, cultural, and institutional. Both economic size and income level of the two countries are important factors affecting trade and are positively correlated, and the economic size has a greater degree of influence than the income level. Geographical distance is an important factor affecting transportation cost, and the closer the geographical distance between the two countries, the lower the transportation cost will be, which is more conducive to enhancing the level of trade between the two countries. Geographical factors are represented by the distance between the capitals of the two countries, the distance by sea, the distance by land, and the presence of a common border. Cultural similarities between two trading parties will greatly facilitate exchanges between the countries, including tourism, culture, and trade. Conversely, cultural differences can hinder exchanges between countries. However, others argue that the promotion of trade by cultural factors is not a simple linear relationship, but an inverted $U$ shape. National culture contains various elements such as language and colonial relations, and language is the most typical representative of the culture. Trade parties who are in an international organization or sign a trade agreement, preferential policies within the organization, and in the trade agreement will enhance the level of trade between the two countries, thus generating a larger trade volume. The analytical model constructed in this study is shown in the following equation:

$$
M=f\left(\mathrm{GDP}_{0}, \mathrm{GDP}_{1}, \mathrm{CON}, \mathrm{LAN}, \mathrm{WTO}\right),
$$

where $M$ denotes the service trade network with the countries along the route. The meaning of other parameters is shown in Table 1.

To avoid the serious multicollinearity between the variables in the traditional measurement method, this study uses the quadratic assignment procedure method to conduct correlation and regression analysis on the factors influencing the service trade export network and value-added trade network of the "Belt and Road" countries. In this study, the QAP model is constructed using the service trade relationship data and trade volume of the countries along the "Belt and Road" as the explanatory variables, and selecting the level of economic development, geographical distance, whether or not the language is the same, whether or not the countries share land borders, and whether or not FTA agreements are signed as the explanatory variables.

The QAP unweighted regression model is given in the following equation:

$$
\begin{aligned}
M(m n) & =\sum_{i=1}^{5} \beta_{i} * P_{i}(m n)+\eta \\
P & \subseteq\left[\mathrm{GDP}_{0}, \mathrm{GDP}_{1}, \mathrm{CON}, \mathrm{LAN}, \mathrm{WTO}\right] .
\end{aligned}
$$

The QAP weighted regression model is given in the following equation:

$$
\begin{aligned}
M(m n)^{w} & =\sum_{i=1}^{5} \beta_{i} * P_{i}^{w_{i}}(m n)+\eta, \\
P & \subseteq\left[\mathrm{GDP}_{0}, \mathrm{GDP}_{1}, \mathrm{CON}, \mathrm{LAN}, \mathrm{WTO}\right] .
\end{aligned}
$$

The actual correlation coefficient results of the unweighted network, the actual correlation coefficient results of the weighted network, the actual value results of the unweighted network, and the actual value results of the weighted network are sorted into a unified result of correlation analysis. In the trade network correlation analysis results, the signs of the variables have not changed in different periods. They are the same as the expected signs, and the significance remains basically unchanged. The fluctuation of the actual correlation coefficient is within the same range. The actual correlation coefficient of the weighted trade network is generally greater than that of the unauthorized trade. The difference in GDP, whether the language is the same, whether the land is bordered, distance, and whether to sign an FTA have significantly affected the formation of service trade relations among countries along the route.

\section{Analysis of Results}

4.1. Analysis of Service Trade Indicators of Countries along the "Belt and Road". From the results of correlation analysis in Figure 2, it can be seen that the results of correlation analysis between weighted and unweighted national service trade networks in 2010-2020 have not changed the sign of the variables in different periods, the same sign change as 
TABle 1: Meaning of parameters.

\begin{tabular}{lccc}
\hline Parameter number & Parameter symbol & Parameter meaning & Expected symbol \\
\hline 1 & $\mathrm{GDP}_{0}$ & Economic scale difference matrix & + \\
2 & $\mathrm{GDP}_{1}$ & Income-level difference matrix & - \\
3 & $\mathrm{GON}$ & Geographic location matrix & + \\
4 & LAN & Language matrix & + \\
5 & WTO & WTO matrix & - \\
\hline
\end{tabular}

expected, the significance remains the same, the fluctuations of the actual correlation coefficients are within the same range, and the actual correlation coefficients of weighted trade networks are generally larger than those of unweighted trade networks. The difference in GDP, whether the language is the same, whether the land is bordering, distance, and whether FTA is signed significantly affect the formation of service trade relations of countries along the route. In the correlation analysis of powerless networks, the variables of GDP difference, language congruence, land bordering, distance, and FTA signing all significantly affect the formation of powerless trade networks in services in the countries along the route from 2010 to 2020. Among them, the GDP difference variables from 2010 to 2020 all passed the 1\% significance level test, and the actual correlation coefficient decreased from 0.155 in 2010 to 0.120 in 2020, a decrease of 0.025 , indicating that the difference in economic level has a positive impact on the formation of national service trade relations, and countries with greater differences in economic level are prone to national service trade relations. In the results of the weighted network correlation analysis, the GDP difference, whether the language is the same, whether the land is bordered, distance, and whether FTA is signed variables are significantly correlated with the changes in the trade volume of the network of service unweighted trade in the countries along the route in 2010-2020. Among them, the GDP difference variables from 2010 to 2020 all pass the significance level test of $5 \%$, and the actual correlation coefficient decreases from 0.154 in 2010 to 0.146 in 2020, a decrease of 0.008 , which is smaller than the decrease in the actual correlation coefficient of the powerless network, indicating that the difference in economic level is positively correlated with the change in the value of service weighted trade network transactions of the countries along the route (see Figure 2).

Using UCINET analysis software, 5000 random permutations were selected to bring the country service unweighted network matrix and weighted network matrix, GDP difference matrix, language binary matrix, bordering binary matrix, distance multivalued matrix, and FTA binary matrix from 2010 to 2020 into the software for regression analysis of the factors influencing the overall network of service trade in the countries along the route, and the values in the table are standardized. The values in the table are the standardized regression coefficients, and the significance level is the significance test result of the standardized regression coefficients. The results are shown in Figure 3. The regression results show that, unlike the QAP correlation analysis, the results of the regression analysis of the weighted national trade in service network and the unweighted trade network from 2010 to 2020 have large differences in the sign, significance, and fluctuations of the standardized regression coefficients of the variables in different periods. In the weighted trade network, the distance variable did not pass the significance test in 2010 and 2012-2020, while the rest of the variables passed the significance test; in the unweighted trade network, variables such as FTA passed the significance test. The GDP difference and FTA variables have the greatest influence on the change in the volume of national trade in services and the formation of trade relations. The standardized regression results of the weighted network regressions from 2010 to 2020 regression coefficients are generally larger than the unweighted network regression results, especially the bordering binary matrix and the FTA binary matrix, which change significantly. The coefficient of determination R2 and the adjustable coefficient of determination are larger than the unweighted network regression results, indicating that variables such as FTA have a strong influence on the weighted network (see Figure 3).

\subsection{Correlation Analysis of Dynamic Evolution Model of} Network Structure. The empirical results in Figure 4 show that the coefficient of GDP is 0.124 , which is significant at a $5 \%$ confidence level, indicating that the importance of a country in the international investment network along the "Belt and Road" is positively influenced by its economic development level, and countries with larger economies tend to enjoy higher international investment status. The coefficient of the gross tax rate is -13.152 , which is also significant at the $5 \%$ confidence level, indicating that gross tax rate hurts a country's position in the network; i.e., countries with higher gross tax rates have less regional importance, and higher gross tax rate means fewer business profits, thus weakening international investment position; while the coefficient of net domestic credit is 3.698, the coefficient of net domestic credit is 3.698 , which is significant at a $1 \%$ confidence level, indicating that the level of banking sector development and financial market development has a significant positive effect on the status and importance of a country, and countries with well-developed financial markets, smooth financing channels, and larger credit volume are easily favored by countries in the region and thus occupy a more important position. The coefficient of urbanization rate $(\mathrm{ft})$ is 16.916 , which means that a higher urbanization rate means better infrastructure, which is conducive to the attractiveness of foreign capital inflow and thus the position in the international direct investment network; the coefficient of the total number of domestic listed companies is 

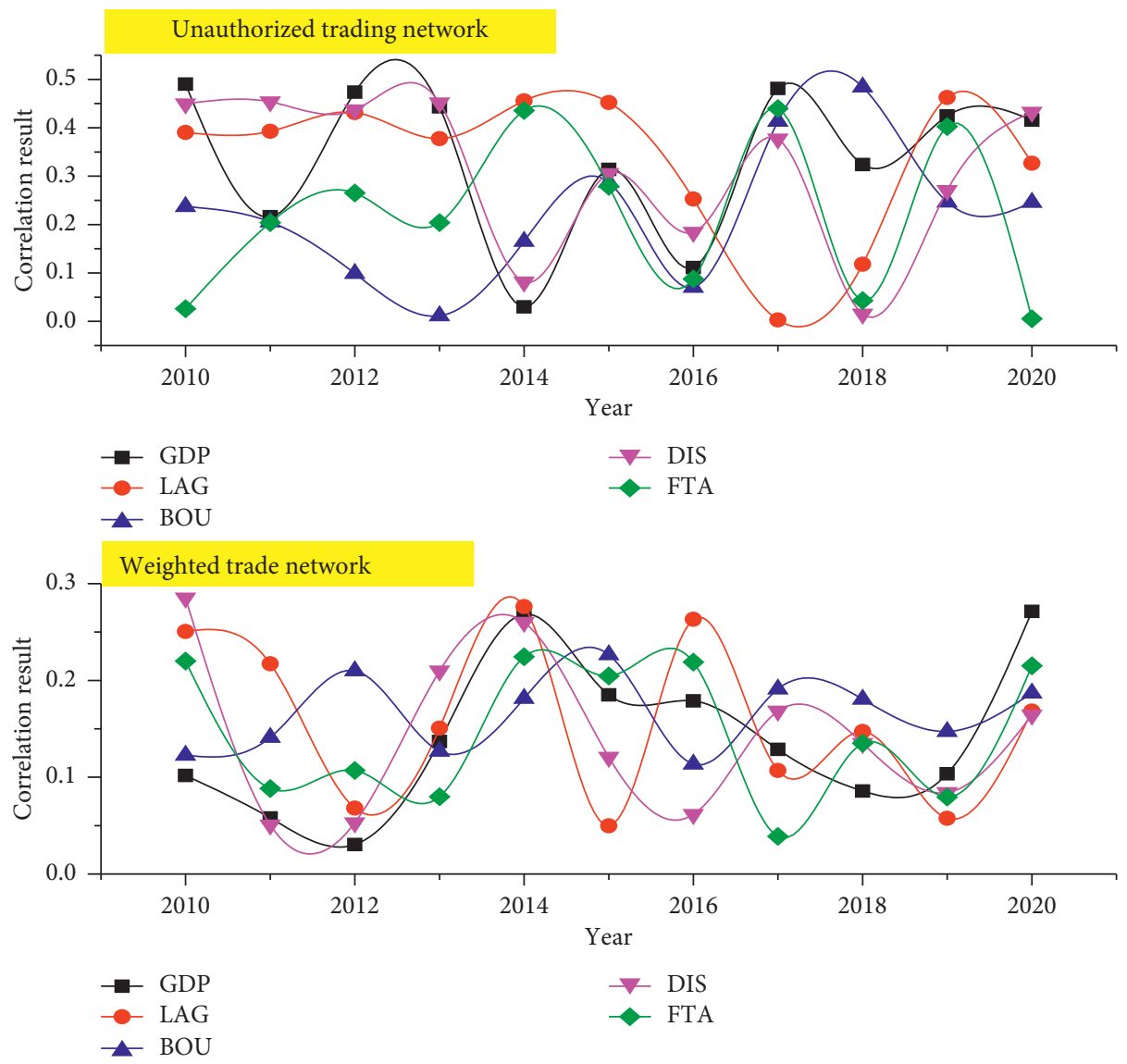

Figure 2: Results of QAP correlation analysis.

small and insignificant, only 0.002, which means that the degree of development of the securities and insurance industry in a country does not have a significant impact on its position in the international direct investment network. Similarly, the coefficient of nonperforming bank loans is also small and insignificant (see Figure 4).

Considering that the research subjects of this study are countries along the "Belt and Road," the grouping test is conducted before and after the "Belt and Road" idea is proposed, and the results are shown in Figure 5.

When the index is greater than 1, it means that the national service trade of the two countries is closely linked, the larger the value, the closer; conversely, it means that the national service trade of the two countries is loosely linked, the smaller the value, the looser. The index of service trade integration with each regional country along the route is calculated and compiled by the index formula. From Figure 6 , it can be seen that the difference between the national service TII index and its development trend with each region is obvious, and the national service trade aggregation index with three regions of Southeast Asia, Central Asia, and Mongolia, and Russia is greater than 1, and the trade connection is close, while the national service trade connection with three regions of West Asia, South Asia, and Central and Eastern Europe is loose. In particular, for each region along the route, the service trade with Southeast Asia has been the strongest and increasingly strong since the official operation of the ASEAN Free Trade Area in 2010; the service trade with Central Asia is unstable but has been in a close state; the service trade with Mongolia and Russia tends to be stable; the service trade with West Asia, Middle East and North Africa, South Asia, Central, and Eastern Europe has been at a low level; and the service trade ties with countries in West Asia, Middle East, North Africa, South Asia, and Central and Eastern Europe have been at a low level (see Figure 6).

\subsection{Analysis of the Impact of Trade in Services in Countries} along the "Belt and Road". Based on the overall level, this study also classifies the 12 service sectors into capital-intensive, industry-intensive, and labor-intensive based on factor intensity. The regression results of the DVA network and FVA network for each industry are presented in Figure 7.

According to Figure 8, it can be seen that the overall dependence on the trade of the "Belt and Road" countries is high, and the countries that have a key dependence on trade include Singapore, Cambodia, Thailand, Indonesia, Malaysia, and the Philippines, most of which are located in Southeast Asia, so Southeast Asian countries are still the economic regions and organizations where trade is more concentrated. Among them, Singapore maintains a continuously growing dependence on the situation, with a 

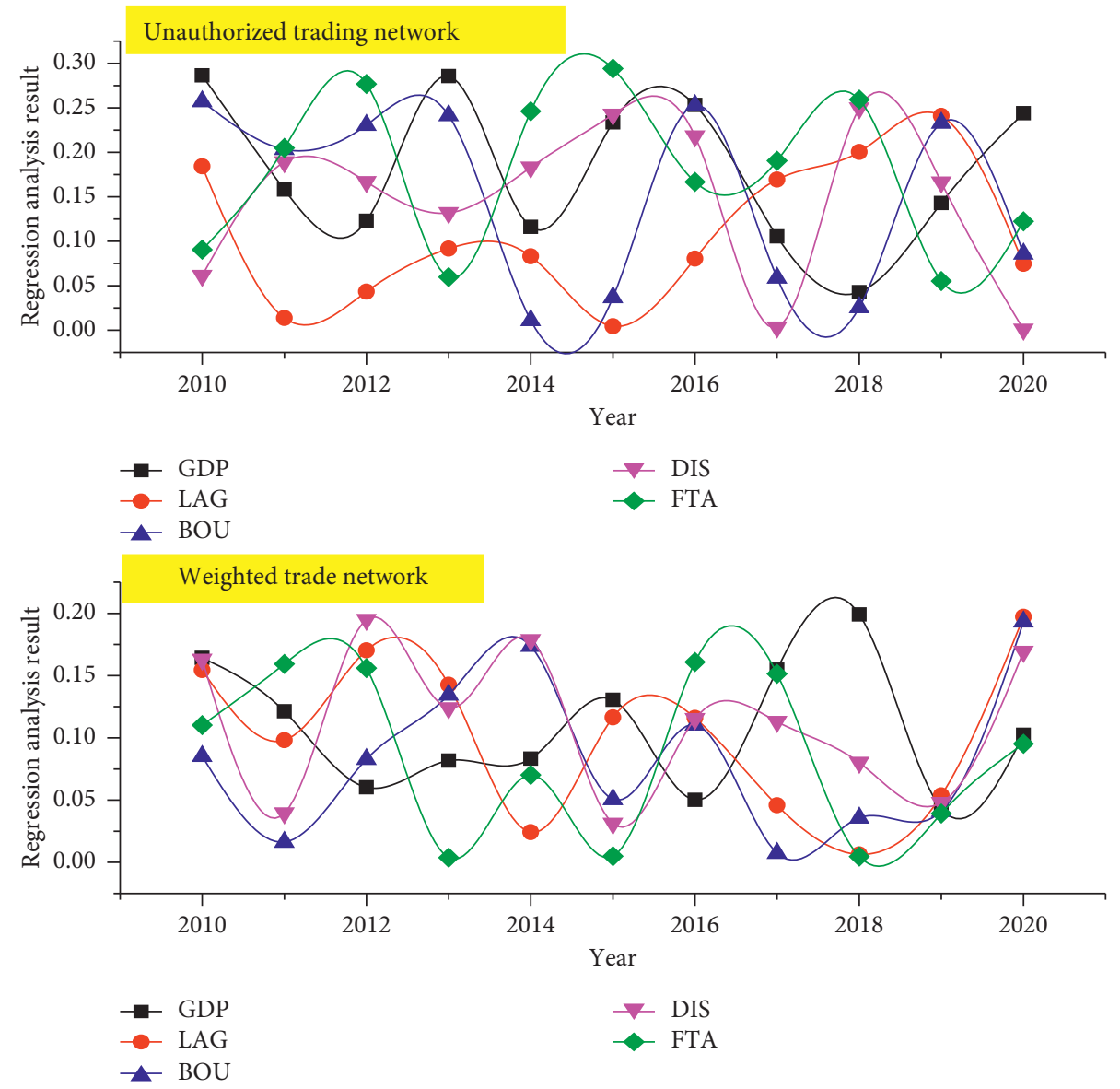

FIgURE 3: Results of QAP regression analysis.

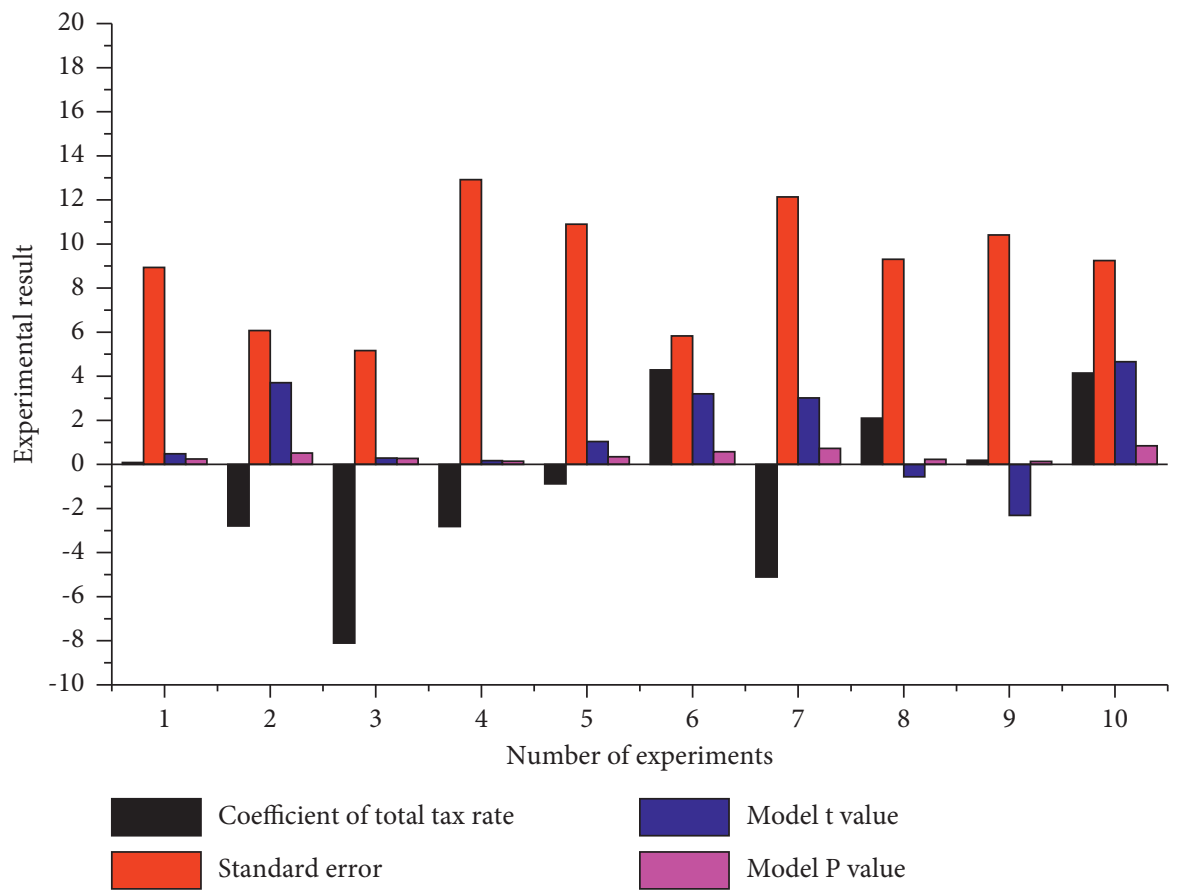

FIGURE 4: Fixed-effects benchmark chess-type results. 

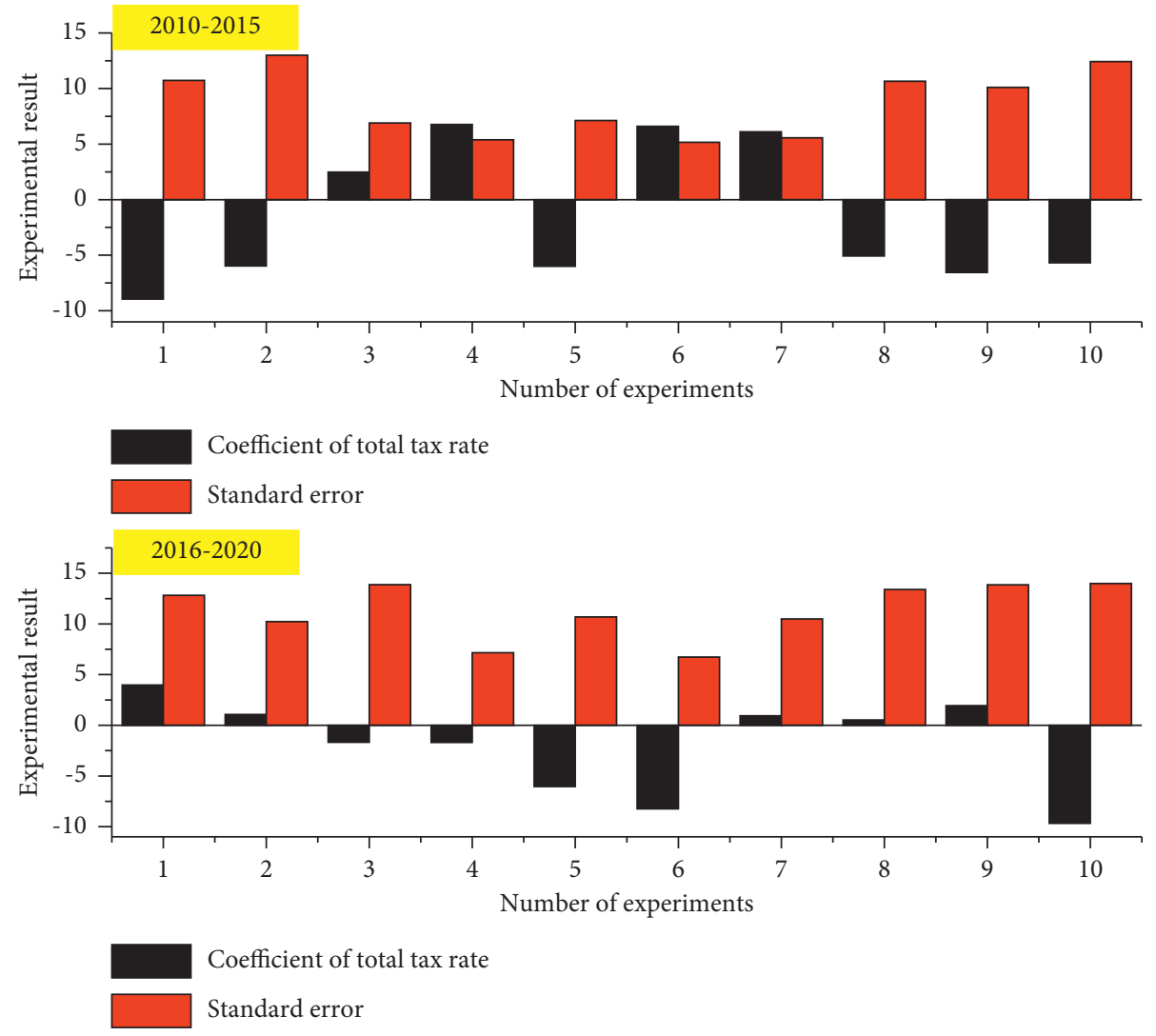

FIgURE 5: Results of the subgroup test.
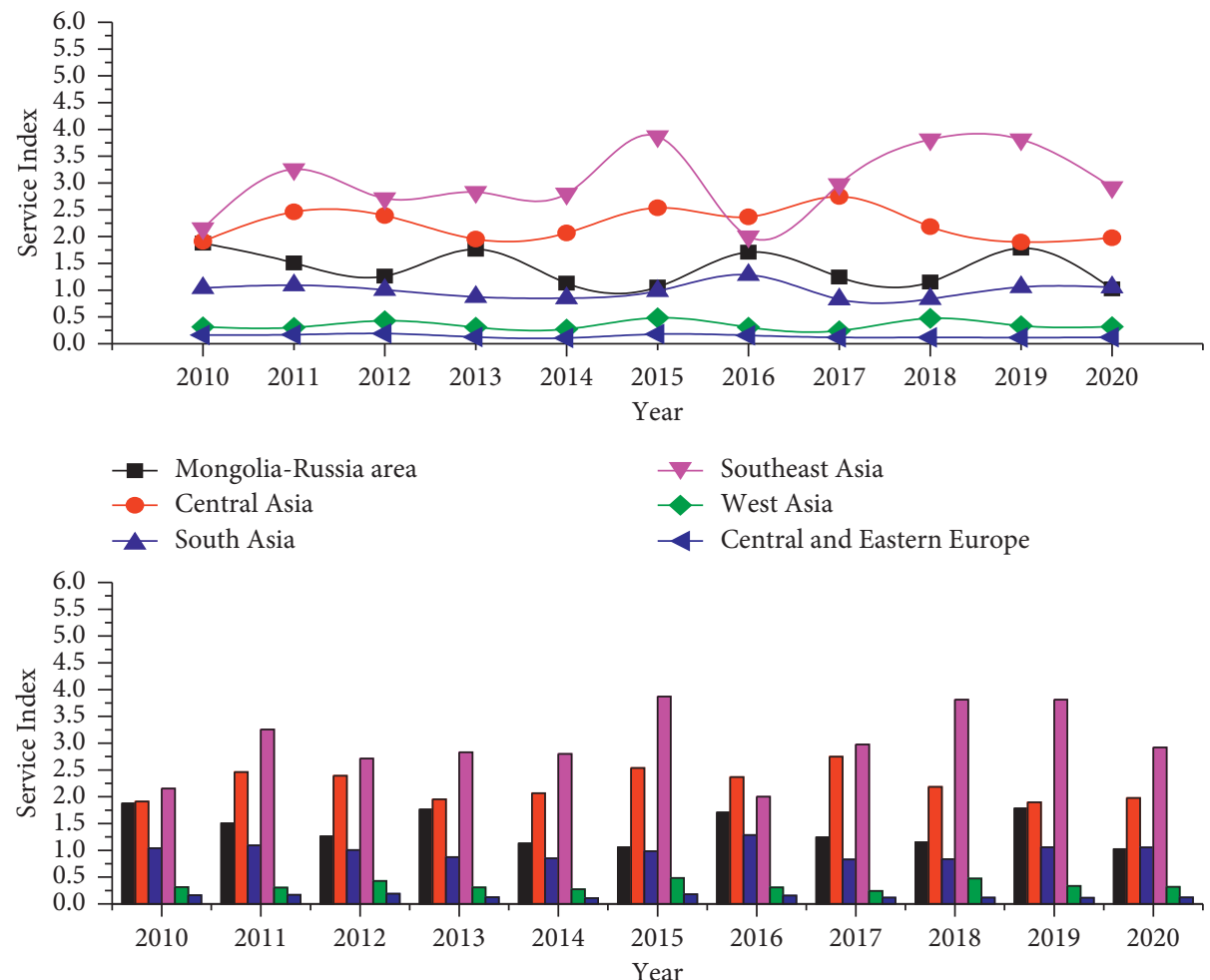

Mongolia-Russia area

$\square$ Southeast Asia

Central Asia

South Asia

West Asia

Central and Eastern Europe

FIgURE 6: Indices of service with countries in each region. 


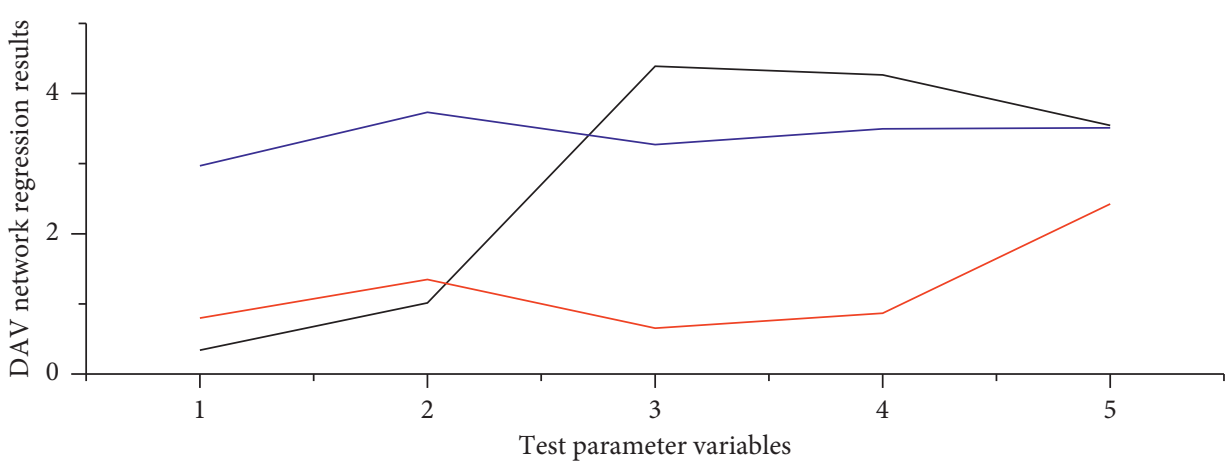

- Capital intensive

Knowledge-intensive

— Labor-intensive

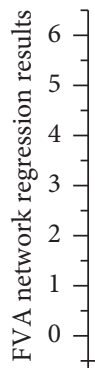

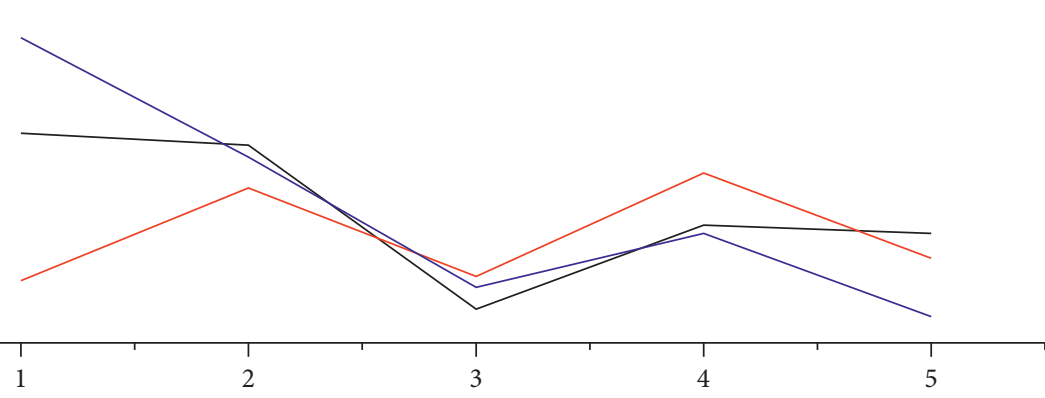

Test parameter variables

- Capital intensive

- Knowledge-intensive

— Labor-intensive

Figure 7: Regression results based on factor intensity breakdown level.
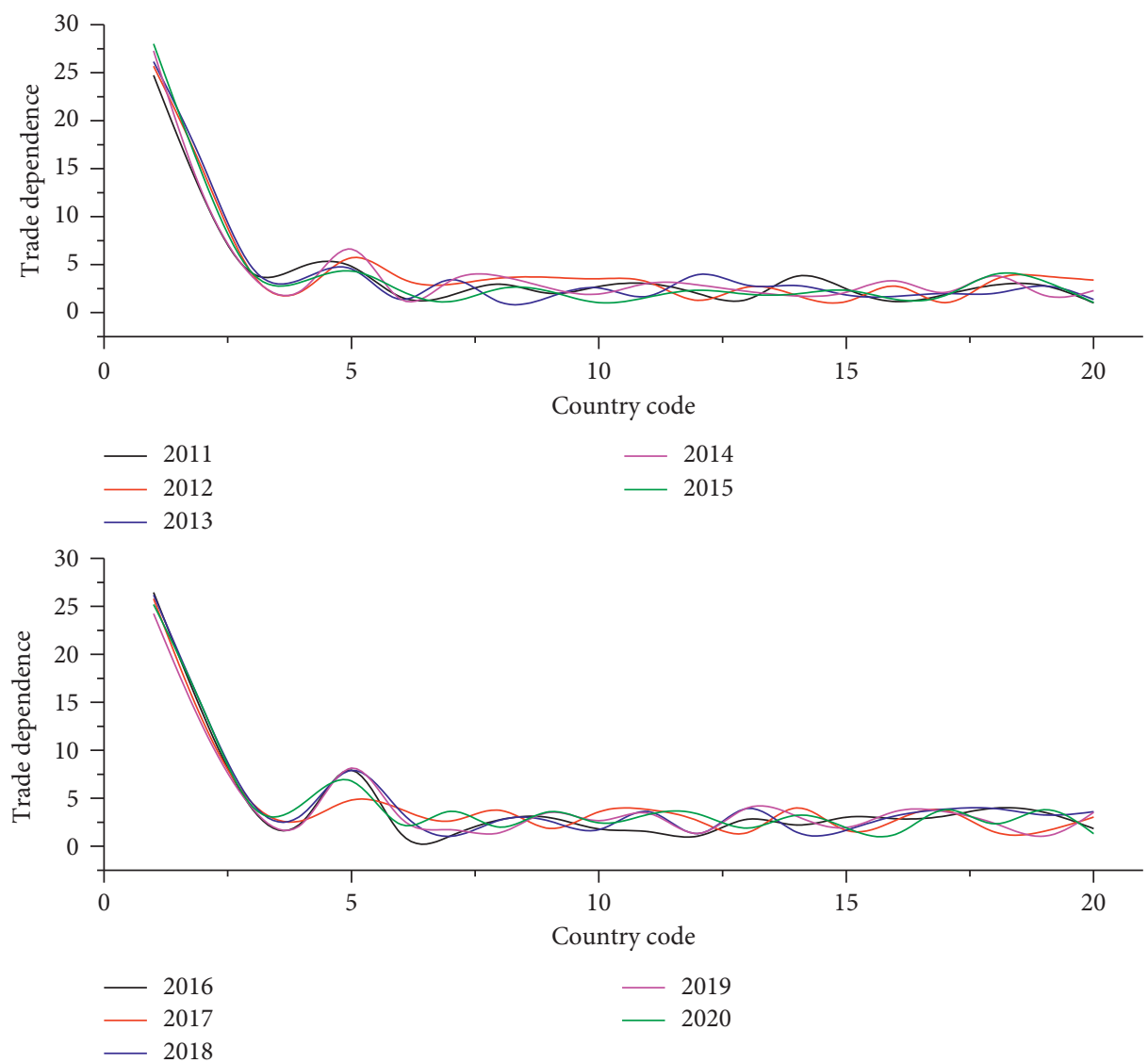

Figure 8: Trade dependence of countries along the "Belt and Road". 
dependence of $24.5 \%$ in 2019 and an increase of $8.12 \%$ over the previous year. Singapore's overall development and economic and cultural are relatively similar, including frequent trade networks between finance, manufacturing, and transportation, the mutual dependence between the two countries is high. Indonesia's economic development has some potential and is an important pivot point for the "One Road" initiative. The Philippines has a high potential for economic growth, but the South Sea dispute has a serious negative impact on the political and economic relations between the two countries. India should focus on cooperation in core areas, such as infrastructure investment, as its backward infrastructure constrains sustainable economic growth and the pressure of domestic and external debt repayment (see Figures 7 and 8).

The construction of the "Belt and Road" regional trade organization is a long-term sustainable development process, not only the beginning, no end. In the process of core countries' participation, they can lead the line with the point, and the line will become the surface to promote the development and construction of the whole strategy. All countries along the route are important participating countries, and the supporting role of the core countries as strategic pivot points is very important. It is necessary to make full use of the cooperative relations of friendly countries to build a prosperous and friendly strategic model and spread the demonstration role. "As there are many countries along the "Belt and Road," each with its color, it is impossible to cover all aspects, and the focus of interaction is different. It is necessary to make full use of friendly countries to establish exemplary cooperation models, to form a mutually beneficial and win-win model, and to drive countries with doubts to actively participate in it.

The larger the economic scale gap between the two countries in the trade network, the easier it is for trade relations to occur and the greater the trade flow. At the same time, the coefficients of variables are larger than those of other variables, indicating that, compared with other variables, the difference in economic scale has the greatest impact on the structure of the unpowered and power trade network. However, the variable coefficients all show a downward trend, indicating that the degree of influence is declining.

\section{Conclusion}

This study combines the strategic background of "Belt and Road" construction, based on the national service trade data of 65 countries along the route from 2010 to 2020, constructs the overall network of service trade of the countries along the route, and analyzes the overall network analysis methods such as network density, average distance, reciprocity, cohesion, centrality, block model, and QAP method.

The overall network density, reciprocity, and cohesion of service trade of the countries along the route have increased by $13.55 \%, 0.26 \%$, and $5.11 \%$, respectively, from 2010 to 2020 , and the overall trend of rapid growth and high values of all three indicates that the countries along with the "Belt and Road" strategy have become more and more connected. This indicates that since the "One Road" strategy has been proposed, the trade in services between countries has become closer and closer, and the degree of interconnection and cohesiveness has been improved. The average distance of the overall service trade network of the countries along the route is about 1.356, which indicates the accessibility of the service trade of the countries along the route.

The construction of the ancient Silk Road, which led to the integration and development of politics, economy, and culture of the countries along the route, shortened the trade distance of the countries along the route and is an important reason for the shorter average distance of the overall network of service trade of the countries along the route. At the same time, the accessibility of trade verifies the necessity of the "Belt and Road" strategy. According to the results of QAP correlation analysis and regression analysis, distance is still an important factor limiting the development of service trade in the countries along the route, so we should play a leading role in strengthening the construction of transportation infrastructure along the "Belt and Road." At the same time, we should accelerate the signing of national service free trade agreements with countries along the route, lower the access threshold, deepen the national service trade cooperation areas, and promote the deep development of national service bilateral trade.

\section{Data Availability}

The data used to support the findings of this study are included within the article.

\section{Conflicts of Interest}

The authors declare that they have no conflicts of interest.

\section{Acknowledgments}

This study was supported by the Social Science Fund: Risk Assessment of Corn lndustry Chain under the Background of Agricultural Supply-side Reform (HB18GL055).

\section{References}

[1] Z. Liao, Z. Wang, and K. Guo, "The dynamic evolution of the characteristics of exchange rate risks in countries along "the belt and road" based on network analysis," PLoS One, vol. 14, no. 9, Article ID e0221874, 2019.

[2] W. Gu and H. Liu, "Spatial structure, hierarchy and formation mechanisms of scientific collaboration networks: evidence of the belt and road regions," Chinese Geographical Science, vol. 30, no. 6, pp. 959-975, 2020.

[3] Z. Liu, T. Wang, J. W. Sonn, and W. Chen, "The structure and evolution of trade relations between countries along the belt and road," Journal of Geographical Sciences, vol. 28, no. 9, pp. 1233-1248, 2018.

[4] J. Chen, J. Huang, L. Zheng, and Z. Chonghui, "An empirical analysis of telecommunication infrastructure promoting the scale of international service trade: based on the panel data of countries along the belt and road," Transformations in Business and Economics, vol. 18, no. 2, pp. 124-139, 2019.

[5] K. Dumor, Y. Li, E. M. Ampaw, C. H. K. Essel, E. O. Essel, and O. M. Mutiiria, "Situating africa in the exports patterns of 
China's belt and road initiative: a network analysis," African Development Review, vol. 33, no. 2, pp. 343-356, 2021.

[6] C. Liu, J. Xu, and H. Zhang, "Competitiveness or complementarity? a dynamic network analysis of international agritrade along the belt and road," Applied Spatial Analysis and Policy, vol. 13, no. 2, pp. 349-374, 2020.

[7] Y. Li, "Influence of the internet on the economic growth of the belt and road region," Global Journal of Emerging Market Economies, vol. 11, no. 3, pp. 248-259, 2019.

[8] S. Saud, S. Chen, A. Danish, and A. Haseeb, "Impact of financial development and economic growth on environmental quality: an empirical analysis from Belt and Road Initiative (BRI) countries," Environmental Science and Pollution Research, vol. 26, no. 3, pp. 2253-2269, 2019.

[9] H. C. Teo, A. M. Lechner, G. W. Walton et al., "Environmental impacts of infrastructure development under the belt and road initiative," Environments, vol. 6, no. 6, pp. 72-90, 2019.

[10] K. Dumor, L. Yao, J. P. Ainam, and W. Ayivi, "Quantitative dynamics effects of belt and road economies trade using structural gravity and neural networks," Sage Open, vol. 11, no. 3, Article ID 21582440211032662, 2021.

[11] T. Summers, "The belt and road initiative in southwest China: responses from yunnan province," The Pacific Review, vol. 34, no. 2, pp. 206-229, 2021.

[12] K. P. Y. Lai, S. Lin, and J. D. Sidaway, "Financing the Belt and Road Initiative (BRI): research agendas beyond the "debttrap" discourse," Eurasian Geography and Economics, vol. 61, no. 2, pp. 109-124, 2020.

[13] K. X. Li, M. Jin, G. Qi, W. Shi, and A. K. Y. Ng, "Logistics as a driving force for development under the belt and road initiative - the Chinese model for developing countries," Transport Reviews, vol. 38, no. 4, pp. 457-478, 2018.

[14] L. Cui and M. Song, "Economic evaluation of the belt and road initiative from an unimpeded trade perspective," International Journal of Logistics Research and Applications, vol. 22, no. 1, pp. 25-46, 2019.

[15] P. T.-W. Lee, Z.-H. Hu, S.-J. Lee, K.-S. Choi, and S.-H. Shin, "Research trends and agenda on the Belt and Road (B\&R) initiative with a focus on maritime transport," Maritime Policy \& Management, vol. 45, no. 3, pp. 282-300, 2018.

[16] Z. Li, Z. Huang, and H. Dong, "The influential factors on outward foreign direct investment: evidence from the "the belt and road," Emerging Markets Finance and Trade, vol. 55, no. 14, pp. 3211-3226, 2019.

[17] C. Ye, S. Li, L. Zhuang, and X. Zhu, "A comparison and case analysis between domestic and overseas industrial parks of China since the belt and road initiative," Journal of Geographical Sciences, vol. 30, no. 8, pp. 1266-1282, 2020.

[18] X. Wang, Y. D. Wong, K. X. Li, and K. F. Yuen, "Transport research under belt and road initiative: current trends and future research agenda," Transportmetrica: Transportation Science, vol. 17, no. 4, pp. 357-379, 2021.

[19] Q. Huang, X. Zhu, C. Liu, W. Wu, F. Liu, and X. Zhang, "Spatialtemporal evolution and determinants of the belt and road initiative: a maximum entropy gravity model approach," Chinese Geographical Science, vol. 30, no. 5, pp. 839-854, 2020.

[20] N. Ramu, V. Pandi, J. D. Lazarus, and S. Radhakrishnan, "A novel trust model for secure group communication in distributed computing," Journal of Organizational and End User Computing, vol. 32, no. 3, pp. 1-14, 2020.

[21] S. S. Gill and A. Shaghaghi, "Security-aware autonomic allocation of cloud resources," Journal of Organizational and End User Computing, vol. 32, no. 3, pp. 15-22, 2020.
[22] H. Zhang, S. Guo, Y. Qian, Y. Liu, and C. Lu, "Dynamic analysis of agricultural carbon emissions efficiency in Chinese provinces along the belt and road," PLoS One, vol. 15, no. 2, Article ID e0228223, 2020.

[23] B. He, "The domestic politics of the belt and road initiative and its implications," Journal of Contemporary China, vol. 28, no. 116, pp. 180-195, 2019. 